\title{
On a new class of $(j, i)$-Symmetric function on conic regions
}

\author{
Saqib Hussain ${ }^{a, *}$, Mohammed Ali Alamri ${ }^{b}$, Maslina Darus ${ }^{b}$ \\ ${ }^{a}$ COMSATS Institute of Information Technology, Abbotabad, Pakistan. \\ ${ }^{b}$ School of Mathematical Sciences, Faculty of Science and Technology, Universiti Kebangsaan Malaysia 43600, Bangi, Selangor, \\ Malaysia.
}

\author{
Communicated by Y. Hu
}

\begin{abstract}
In this article, a new class of functions is defined using the concepts of $(\mathfrak{j}, \mathfrak{i})$-symmetric functions and Janowski functions in conic regions. Certain interesting coefficient inequalities are discussed. (c)2017 All rights reserved.
\end{abstract}

Keywords: Analytic functions, subordination, conic domain, symmetric functions.

2010 MSC: 30C45, 30C50.

\section{Introduction and preliminaries}

Let $\mathcal{A}$ represent the class of analytic functions $f$ defined in the unit disc $E=\{z:|z|<1\}$ and given by

$$
f(z)=z+\sum_{n=2}^{\infty} a_{n} z^{n} .
$$

Also let $S$, be the well-known subclass of $\mathcal{A}$ consisting of functions which are univalent. An analytic function $f$ is said to be subordinate to another analytic function $g$, written as $f \prec g$, if there exists a Schwarz function $w$ which is analytic in $E$ with $w(0)=0$ and $|w(z)|<1$, for all $z \in E$, such that $f(z)=g(w(z)), z \in E$. If $g$ is univalent in $E$ then $f \prec g$ if and only if $f(0)=g(0)$ and $f(E) \subset g(E)$.

Let $\mathrm{P}$ denote the class of analytic functions of the form

$$
p(z)=1+\sum_{n=1}^{\infty} p_{n} z^{n},
$$

defined on unit disk $E$ and satisfying $p(0)=1$ and $\operatorname{Re}\{p(z)\}>0$, for all $z \in E$. See [3] for more details on class P.

Let $P[A, B]$ denote the class of analytic functions $p$ defined on $E$ with the representation

$$
p(z)=\frac{1+A w(z)}{1+B w(z)}
$$

\footnotetext{
*Corresponding author

Email addresses: saqib_math@yahoo.com (Saqib Hussain), alamri62612@gmail.com (Mohammed Ali Alamri), maslina@ukn. edu.my (Maslina Darus)

doi:10.22436/jnsa.010.09.06
}

Received 2017-02-09 
where $w(0)=0,|w(z)|<1$. We say that $p \in P[A, B]$ if and only if

$$
p(z) \prec \frac{1+A z}{1+B z}
$$

where $-1 \leqslant B<A \leqslant 1$. Geometrically, a function $p(z) \in P[A, B]$ maps the open unit disk $E$ onto the disk defined by the domain

$$
\Omega[A, B]=\left\{w:\left|w-\frac{1-A B}{1-B^{2}}\right|<\frac{1-A B}{1-B^{2}}\right\} .
$$

The relation between the classes $\mathrm{P}$ and $\mathrm{P}[\mathrm{A}, \mathrm{B}]$ is expressed as follows:

$$
p(z) \in P \Leftrightarrow \frac{(A+1) p(z)-(A-1)}{(B+1) p(z)-(B-1)} \in P[A, B] .
$$

The class $P[A, B]$ was introduced by Janowski [4], see also [12]. Kanas and Wisniowska [7] introduced and studied the class of k-uniformly convex functions denoted by k- UCV and the class of k-starlike functions denoted by k-ST. These classes were defined subject to the conic region $\Omega_{k}$ given by

$$
\Omega_{\mathrm{k}}=\left\{u+\boldsymbol{I} v: u>k \sqrt{(u-1)^{2}+v^{2}}, u>0\right\},
$$

where $k \in[0, \infty)$ is a parameter and its suitable values represent right half plane for $k=0$, hyperbola for $0<k<1$, a parabola for $k=1$ and ellipse for $k>1$.

Kanas and Wisniowska [7] showed that the extremal functions $p_{k}(z)$ for conic regions are convex univalent and given by

$$
p_{k}(z)= \begin{cases}\frac{1+z}{1-z^{\prime}} & k=0, \\ 1+\frac{2}{1-k^{2}}\left[\frac{2}{\pi}(\arccos k) \operatorname{arctanh} \sqrt{z}\right], & 0<k<1, \\ 1+\frac{2}{\pi^{2}}\left(\log \frac{1+\sqrt{z}}{1-\sqrt{z}}\right)^{2}, & k=1, \\ 1+\frac{2}{k^{2}-1}\left[\sin \left(\frac{\pi}{2 R(t)} \int_{0}^{\frac{u}{\sqrt{t}}} \frac{1}{\sqrt{1-x^{2}} \sqrt{1-(t x)^{2}}} d x\right)-1\right], & k>1,\end{cases}
$$

where $R(t)$ is the Legendre's complete elliptic integral of the first kind with $R^{\prime}(t)=\sqrt{1-t^{2}}$ as its complementary integral, $u(z)=\frac{z-\sqrt{t}}{1-\sqrt{t} z}, t \in(0,1)$ and $z \in E$ is chosen in such a way that $k=\cosh \left(\frac{\pi R^{\prime}(t)}{R(t)}\right)$.

The function $p_{k}(z)$ in [5] is given as $p_{k}(z)=1+\delta_{1} z+\delta_{2} z^{2}+\cdots$, where

$$
\delta_{k}= \begin{cases}\frac{8(\arccos (k))^{2}}{\pi^{2}\left(1-k^{2}\right)}, & 0<k<1, \\ \frac{8}{\pi^{2}}, & k=1 \\ \frac{\pi^{2}}{4 \sqrt{t}\left(k^{2}-1\right) R^{2}(t)(1+t)}, & k>1 .\end{cases}
$$

A function $p$ is said to be in the class $k-P[A ; B], 1 \leqslant B<A \leqslant 1, k \geqslant 0$, if and only if,

$$
p(z) \prec \frac{(A+1) p_{k}(z)-(A-1)}{(B+1) p_{k}(z)-(B-1)}
$$


where $p_{k}(z)$ is defined in (1.2), see [10].

Let $i$ be a positive integer. A domain $D$ is said to be $i$-fold symmetric if a rotation of $D$ about the origin through an angle $2 \pi / i$ carries $D$ onto itself. A function $f$ is said to be $i$-fold symmetric in $E$ if for every $z$ in $E$

$$
f\left(e^{2 \pi I / i} z\right)=e^{2 \pi I / i} f(z),
$$

where $I$ represents the imaginary unit. $i$-fold symmetric functions are denoted by $S^{(i)}$ and for $i=2$ we get the class of the odd univalent functions. The notion of $(j, i)$-symmetrical functions $(i=2,3, \cdots ; j=$ $0,1,2, \cdots, i-1)$ is a generalization of the notions of even, odd, $i$-symmetrical functions and also generalizes the well-known result that each function defined on a symmetrical subset can be uniquely expressed as the sum of an even function and an odd function. The theory of $(j, i)$-symmetrical functions has many interesting applications, for instance in the investigation of the set of fixed points of mappings, for the estimation of the absolute value of some integrals, and for obtaining some results of the type of certain uniqueness theorem for holomorphic mappings [9].

For $\varepsilon=\exp \left(\frac{2 \pi I}{i}\right),(i=2,3, \cdots ; j=0,1,2, \cdots, i-1)$, where $i \geqslant 2$ is a natural number, a function $f: E \rightarrow \mathbb{C}$ is called $(j, i)$-symmetric if

$$
f\left(\varepsilon^{j} z\right)=\varepsilon^{j} f(z), z \in E,
$$

the family of all $(j, i)$-symmetrical functions is denoted be $S^{(j, i)}$. Also $S^{(0,2)}, S^{(1,2)}$, and $S^{(1, i)}$ are called even, odd, and $i$-symmetric functions respectively. For Janowski type functions associated with $k$ symmetric points, see [8].

We have the following decomposition theorem.

Theorem 1.1 ([9]). For every mapping $\mathrm{f}: \mathrm{E} \rightarrow \mathbb{C}$ and $\mathrm{D}$ being an $\mathrm{i}$-fold symmetric set, there exists exactly a unique sequence of $(j, i)$-symmetrical functions $\mathrm{f}_{j, i}(z)$ as in the form below

$$
f(z)=\sum_{j=0}^{i-1} f_{j, i}(z)
$$

From (1.4), we have

$$
f_{j, i}(z)=\frac{1}{i} \sum_{\nu=0}^{i-1} \varepsilon^{-v j} f\left(\varepsilon^{v} z\right)=\frac{1}{i} \sum_{v=0}^{i-1} \varepsilon^{-v j}\left(\sum_{n=1}^{\infty} a_{n}\left(\varepsilon^{v} z\right)^{n}\right),
$$

then

$$
f_{j, i}(z)=\sum_{n=1}^{\infty} \psi_{n} a_{n} z^{n}, a_{1}=1
$$

where $\varepsilon=\exp \left(\frac{2 \pi I}{i}\right),(i=2,3, \cdots ; j=0,1,2, \cdots, i-1)$, where $i \geqslant 2$ is a natural number and for $l \in \mathbb{N}_{0}$

$$
\psi_{n}=\frac{1}{i} \sum_{v=0}^{i-1} \varepsilon^{(n-j) v}= \begin{cases}1, & n=l i+j \\ 0, & n \neq l i+j\end{cases}
$$

where $(f \in \mathcal{A}, i=1,2, \cdots ; j=0,1,2, \cdots i-1)$.

Al-Sarari and Latha [2] defined the following class using the concepts $(j, i)$-symmetric functions. A function $f$ is said to be in the class $k-V^{(j, i)}[A, B], k \geqslant 0,-1 \leqslant B<A \leqslant 1$, if and only if

$$
\operatorname{Re}\left(\frac{(B-1) \frac{z f^{\prime}(z)}{f_{j, i}(z)}-(A-1)}{(B+1) \frac{z f^{\prime}(z)}{f_{j, i}(z)}-(A+1)}\right)>k\left|\frac{(B-1) \frac{z f^{\prime}(z)}{f_{j, i}(z)}-(A-1)}{(B+1) \frac{z f^{\prime}(z)}{f_{j, i}(z)}-(A+1)}-1\right| .
$$


Lemma 1.2 ([11]). Let $h(z)=1+\sum_{n=1}^{\infty} c_{n} z^{n} \in P[A, B]$. Then

$$
\left|c_{n}\right| \leqslant\left|\delta_{A B}\right|,\left|\delta_{A B}\right|=\frac{(A-B)\left|\delta_{k}\right|}{2},
$$

where $\delta_{\mathrm{k}}$ is defined by (1.3).

The purpose of this paper is to define a new class of functions by using the concepts of $(j, i)$-symmetric functions and Janowski functions in conic regions. Certain interesting coefficient inequalities are also discussed.

\section{Main results}

Let us now extend the idea of Al-Sarari and Latha [2] to define the class $k-G^{(j, i)}[\lambda, A, B]$.

2.1. The class $k-\mathrm{G}^{(\mathrm{j}, \mathrm{i})}[\lambda, \mathrm{A}, \mathrm{B}]$

A function $f$ is said to be in the class $k-G^{(j, i)}[\lambda, A, B], k \geqslant 0,-1 \leqslant B<A \leqslant 1$, if and only if

$$
\operatorname{Re}\left(\frac{(B-1) \frac{z f^{\prime}(z)+\lambda z^{2} f^{\prime \prime}(z)}{f_{j, i}(z)}-(A-1)}{(B+1) \frac{z f^{\prime}(z)+\lambda z^{2} f^{\prime \prime}(z)}{f_{j, i}(z)}-(A+1)}\right)>k\left|\frac{(B-1) \frac{z f^{\prime}(z)+\lambda z^{2} f^{\prime \prime}(z)}{f_{j, i}(z)}-(A-1)}{(B+1) \frac{z f^{\prime}(z)+\lambda z^{2} f^{\prime \prime}(z)}{f_{j, i}(z)}-(A+1)}-1\right| .
$$

For $\lambda=0$ we have the following special cases of the class $k-G^{(j, i)}[\lambda, A, B]$ :

(i) $k-G^{(1, i)}[0, A, B]=k-S T[A, B, i]$, the classes introduced by Al-Sarari and Latha [1];

(ii) $k-G^{(1,1)}[0, A, B]=k-S T[A, B]$, the classes introduced by Noor and Malik [11];

(iii) $k-G^{(1,1)}[0,1,-1]=k-S T$, the well-known classes introduced by Kanas and Wisniowska [6].

Theorem 2.1. A function $\mathrm{f} \in \mathcal{A}$ and of the form

$$
f(z)=z+\sum_{n=2}^{\infty} a_{n} z^{n}
$$

is in the class $k-G^{(j, i)}[\lambda, A, B]$ if it satisfies the condition

$$
\begin{gathered}
\sum_{n=2}^{\infty}\left[2(k+1)\left|\psi_{n}-n-\lambda n\right|+\left|n(B+1)+\lambda(B+1) n(n-1)-(A+1) \psi_{n}\right|\right]\left|a_{n}\right| \\
\quad<\left|(B+1)-(A+1) \psi_{n}\right|-2(k+1)\left|\psi_{n}-1\right|
\end{gathered}
$$

$k \geqslant 0,-1 \leqslant B<A \leqslant 1$ and $\psi_{n}$ is defined in (1.5).

Proof. As

$$
\operatorname{Re}\left(\frac{(B-1) \frac{z f^{\prime}(z)+\lambda z^{2} f^{\prime \prime}(z)}{f_{j, i}(z)}-(A-1)}{(B+1) \frac{z f^{\prime}(z)+\lambda z^{2} f^{\prime \prime}(z)}{f_{j, i}(z)}-(A+1)}\right)>k\left|\frac{(B-1) \frac{z f^{\prime}(z)+\lambda z^{2} f^{\prime \prime}(z)}{f_{j, i}(z)}-(A-1)}{(B+1) \frac{z f^{\prime}(z)+\lambda z^{2} f^{\prime \prime}(z)}{f_{j, i}(z)}-(A+1)}-1\right|,
$$

we have

$$
\operatorname{Re}\left(\frac{(B-1) \frac{z f^{\prime}(z)+\lambda z^{2} f^{\prime \prime}(z)}{f_{j, i}(z)}-(A-1)}{(B+1) \frac{z f^{\prime}(z)+\lambda z^{2} f^{\prime \prime}(z)}{f_{j, i}(z)}-(A+1)}+1-1\right)>k\left|\frac{(B-1) \frac{z f^{\prime}(z)+\lambda z^{2} f^{\prime \prime}(z)}{f_{j, i}(z)}-(A-1)}{(B+1) \frac{z f^{\prime}(z)+\lambda z^{2} f^{\prime \prime}(z)}{f_{j, i}(z)}-(A+1)}-1\right|,
$$




$$
k\left|\frac{(B-1) \frac{z f^{\prime}(z)+\lambda z^{2} f^{\prime \prime}(z)}{f_{j, i}(z)}-(A-1)}{(B+1) \frac{z f^{\prime}(z)+\lambda z^{2} f^{\prime \prime}(z)}{f_{j, i}(z)}-(A+1)}-1\right|-\operatorname{Re}\left(\frac{(B-1) \frac{z f^{\prime}(z)+\lambda z^{2} f^{\prime \prime}(z)}{f_{j, i}(z)}-(A-1)}{(B+1) \frac{z f^{\prime}(z)+\lambda z^{2} f^{\prime \prime}(z)}{f_{j, i}(z)}-(A+1)}-1\right)<1 .
$$

\section{Consider L.H.S}

$$
\begin{aligned}
& \leqslant k\left|\frac{(B-1) \frac{z f^{\prime}(z)+\lambda z^{2} f^{\prime \prime}(z)}{f_{j, i}(z)}-(A-1)}{(B+1) \frac{z f^{\prime}(z)+\lambda z^{2} f^{\prime \prime}(z)}{f_{j, i}(z)}-(A+1)}-1\right|+\left|\frac{(B-1) \frac{z f^{\prime}(z)+\lambda z^{2} f^{\prime \prime}(z)}{f_{j, i}(z)}-(A-1)}{(B+1) \frac{z f^{\prime}(z)+\lambda z^{2} f^{\prime \prime}(z)}{f_{j, i}(z)}-(A+1)}-1\right| \\
& =(k+1)\left|\frac{(B-1)\left[z f^{\prime}(z)+\lambda z^{2} f^{\prime \prime}(z)\right]-(A-1) f_{j, i}(z)}{(B+1)\left[z f^{\prime}(z)+\lambda z^{2} f^{\prime \prime}(z)\right]-(A+1) f_{j, i}(z)}-1\right| \\
& =(k+1) \mid \frac{(B-1)\left[z f^{\prime}(z)+\lambda z^{2} f^{\prime \prime}(z)\right]-(A-1) f_{j, i}(z)}{(B+1)\left[z f^{\prime}(z)+\lambda z^{2} f^{\prime \prime}(z)\right]-(A+1) f_{j, i}(z)} \\
& -\frac{(B+1)\left[z f^{\prime}(z)+\lambda z^{2} f^{\prime \prime}(z)\right]-(A+1) f_{j, i}(z)}{(B+1)\left[z f^{\prime}(z)+\lambda z^{2} f^{\prime \prime}(z)\right]-(A+1) f_{j, i}(z)} \mid \\
& =(k+1)\left|\frac{-2\left[z f^{\prime}(z)+\lambda z^{2} f^{\prime \prime}(z)\right]+2 f_{j, i}(z)}{(B+1)\left[z f^{\prime}(z)+\lambda z^{2} f^{\prime \prime}(z)\right]-(A+1) f_{j, i}(z)}\right| \\
& =2(k+1)\left|\frac{f_{j, i}(z)-\left[z f^{\prime}(z)+\lambda z^{2} f^{\prime \prime}(z)\right]}{(B+1)\left[z f^{\prime}(z)+\lambda z^{2} f^{\prime \prime}(z)\right]-(A+1) f_{j, i}(z)}\right| .
\end{aligned}
$$

Now, observe that

$$
z f^{\prime}(z)=z+\sum_{n=2}^{\infty} n a_{n} z^{n}
$$

and

$$
z^{2} f^{\prime \prime}(z)=\sum_{n=2}^{\infty} n(n-1) a_{n} z^{n}
$$

Putting (1.1), (2.3), and (2.4) in (2.2), we get

$$
\begin{aligned}
& =2(k+1)\left|\frac{\sum_{n=1}^{\infty} \psi_{n} a_{n} z^{n}-\left[z+\sum_{n=2}^{\infty} n a_{n} z^{n}+\lambda \sum_{n=2}^{\infty} n(n-1) a_{n} z^{n}\right]}{(B+1)\left[z+\sum_{n=2}^{\infty} n a_{n} z^{n}+\lambda \sum_{n=2}^{\infty} n(n-1) a_{n} z^{n}\right]-(A+1) \sum_{n=1}^{\infty} \psi_{n} a_{n} z^{n}}\right| \\
& =2(k+1)\left|\frac{\psi_{1} z+\sum_{n=2}^{\infty} \psi_{n} a_{n} z^{n}-z-\sum_{n=2}^{\infty} n a_{n} z^{n}-\lambda \sum_{n=2}^{\infty} n(n-1) a_{n} z^{n}}{(B+1)\left[z+\sum_{n=2}^{\infty} n a_{n} z^{n}+\lambda \sum_{n=2}^{\infty} n(n-1) a_{n} z^{n}\right]-(A+1) \psi_{1} z-(A+1) \sum_{n=2}^{\infty} \psi_{n} a_{n} z^{n}}\right| \\
& =2(k+1)\left|\frac{\left(\psi_{1}-1\right) z+\sum_{n=2}^{\infty}\left[\psi_{n}-n-\lambda n(n-1)\right] a_{n} z^{n}}{\left[(B+1)-(A+1) \psi_{1}\right] z+\sum_{n=2}^{\infty}\left[(B+1) n+\lambda(B+1) n(n-1)-(A+1) \psi_{n}\right] a_{n} z^{n}}\right| .
\end{aligned}
$$

Now, using triangle inequality we have

$$
\leqslant 2(k+1)\left[\frac{\left|\psi_{1}-1\right|+\sum_{n=2}^{\infty}\left|\psi_{n}-n-\lambda n(n-1)\right|\left|a_{n}\right|}{\left|(B+1)-(A+1) \psi_{1}\right|-\sum_{n=2}^{\infty}(B+1) n+\lambda(B+1) n(n-1)-(A+1) \psi_{n}\left|a_{n}\right|}\right] .
$$

The last expression is bounded by 1 , then

$$
\begin{aligned}
& \sum_{n=2}^{\infty}\left[2(k+1)\left|\psi_{n}-n-\lambda n(n-1)\right|+\left|(B+1) n+\lambda(B+1) n(n-1)-(A+1) \psi_{n}\right|\right]\left|a_{n}\right| \\
& \quad<\left|(B+1)-(A+1) \psi_{1}\right|-2(k+1)\left|\psi_{1}-1\right|
\end{aligned}
$$

which completes the proof.

For $\lambda=0$ and $j=1$ in the above theorem we get the following result given by [1]. 
Corollary 2.2. A function $\mathrm{f} \in \mathcal{A}$ and of the form (1.1) is in the class $\mathrm{k}-\mathrm{ST}[\mathrm{A}, \mathrm{B}, \mathrm{N}]$ if it satisfies the following inequality

$$
\sum_{n=2}^{\infty}\left[2(k+1)\left(n-\sigma_{N}(n)\right)+|(B+1) n-(A+1)|\right]\left|a_{n}\right|<|(B-A)|,
$$

where $\sigma_{\mathrm{N}}(\mathrm{n})$ is given by

$$
\sigma_{N}(n)= \begin{cases}1, & \text { if } n=l N+1, \\ 0, & \text { if } n \neq l N+1\end{cases}
$$

and $-1 \leqslant \mathrm{~B}<\mathrm{A} \leqslant 1$ and $\mathrm{k} \geqslant 0$.

Putting $j=i=1$ and $\lambda=0$, we have the following known results proved by Noor and Malik [11].

Corollary 2.3. A function $\mathrm{f} \in \mathcal{A}$ and of the form (1.1) is in the class k-ST[A, B] if it satisfies the condition

$$
\sum_{n=2}^{\infty}[2(k+1)(n-1)|n(B+1)-(A+1)|]\left|a_{n}\right|<|B-A|,
$$

where $-1 \leqslant B<A \leqslant 1$ and $k \geqslant 0$.

For $j=i=A=-B=1$ and $\lambda=0$, we have the following results given by Kanas and Wisniowska [6].

Corollary 2.4. A function $\mathrm{f} \in \mathcal{A}$ and expressed in form (1.1) is in the class $\mathrm{k}-\mathrm{ST}$, if it satisfies the condition

$$
\sum_{n=2}^{\infty}[n+k(n-1)]\left|a_{n}\right|<1 .
$$

Theorem 2.5. A function $\mathrm{f} \in \mathcal{A}$ and of the form

$$
f(z)=z+\sum_{n=2}^{\infty} a_{n} z^{n}
$$

is in the class $k-G^{(j, i)}[\lambda, A, B]$ if it satisfies the condition

$$
\left|a_{n}\right| \leqslant \prod_{h=1}^{n-1} \frac{\left|\delta_{k}(A-B)-2\left(h-\psi_{h}-\lambda h(h-1)\right) B\right|}{2\left(h+1-\psi_{h+1}-\lambda h(h-1)\right)},
$$

where $n \geqslant 2, k \geqslant 0,-1 \leqslant B<A \leqslant 1$ and $\delta_{k}, \psi_{n}$ are defined respectively in (1.3) and (1.5).

Proof. By definition (2.1)

$$
\frac{z f^{\prime}(z)+\lambda z^{2} f^{\prime \prime}(z)}{f_{j, i}(z)}=p(z)
$$

where

$$
p(z) \in k-P[A, B],
$$

then we have the following expression

$$
z f^{\prime}(z)+\lambda z^{2} f^{\prime \prime}(z)=f_{j, i}(z) p(z) .
$$

Since $p(z)=1+\sum_{n=1}^{\infty} c_{n} z^{n}$, therefore we have

$$
z f^{\prime}(z)+\lambda z^{2} f^{\prime \prime}(z)=f_{j, i}(z)\left(1+\sum_{n=1}^{\infty} c_{n} z^{n}\right) .
$$


Now

$$
f^{\prime}(z)=1+\sum_{n=2}^{\infty} n a_{n} z^{n-1} \Rightarrow z f^{\prime}(z)=z+\sum_{n=2}^{\infty} n a_{n} z^{n}
$$

We know that

$$
f^{\prime \prime}(z)=\sum_{n=2}^{\infty} n(n-1) a_{n} z^{n-2} \Rightarrow z^{2} f^{\prime \prime}(z)=\sum_{n=2}^{\infty} n(n-1) a_{n} z^{n} .
$$

Also

$$
f_{j, i}(z)=\sum_{n=1}^{\infty} \psi_{n} a_{n} z^{n}, a_{1}=1 .
$$

Thus, we get the following equations

$$
\begin{aligned}
& z+\sum_{n=2}^{\infty} n a_{n} z^{n}+\lambda \sum_{n=2}^{\infty} n(n-1) a_{n} z^{n}=\sum_{n=1}^{\infty} \psi_{n} a_{n} z^{n}\left(1+\sum_{n=1}^{\infty} c_{n} z^{n}\right), \\
& z+\sum_{n=2}^{\infty} n a_{n} z^{n}+\lambda \sum_{n=2}^{\infty} n(n-1) a_{n} z^{n}=\sum_{n=1}^{\infty} \psi_{n} a_{n} z^{n}+\sum_{n=1}^{\infty} \psi_{n} a_{n} z^{n} \sum_{n=1}^{\infty} c_{n} z^{n}, \\
& \left(1-\psi_{1}\right) z+\sum_{n=2}^{\infty} n a_{n} z^{n}+\lambda \sum_{n=2}^{\infty} n(n-1) a_{n} z^{n}-\sum_{n=2}^{\infty} \psi_{n} a_{n} z^{n}=\sum_{n=1}^{\infty} \psi_{n} a_{n} z^{n} \sum_{n=1}^{\infty} c_{n} z^{n}, \\
& \left(1-\psi_{1}\right) z+\sum_{n=2}^{\infty}\left[n+\lambda n(n-1)-\psi_{n}\right] a_{n} z^{n}=\sum_{n=1}^{\infty} \psi_{n} a_{n} z^{n} \sum_{n=1}^{\infty} c_{n} z^{n} .
\end{aligned}
$$

By using Cauchy product formula on R.H.S above we get

$$
\left(1-\psi_{1}\right) z+\sum_{n=2}^{\infty}\left[n+\lambda n(n-1)-\psi_{n}\right] a_{n} z^{n}=\sum_{n=1}^{\infty}\left[\sum_{h=0}^{n-1} \psi_{h} a_{h} c_{n-h}\right] z^{n} .
$$

By equating coefficients of $z^{\mathfrak{n}}$ in the last expression above, we have

$$
\left[n+\lambda n(n-1)-\psi_{n}\right] a_{n}=\sum_{h=0}^{n-1} \psi_{h} a_{h} c_{n-h}, \quad a_{1}=\psi_{1}=1 .
$$

Taking modulus of the above equation we have

$$
\left[n+\lambda n(n-1)-\psi_{n}\right]\left|a_{n}\right| \leqslant \sum_{h=0}^{n-1} \psi_{h}\left|a_{h}\right|\left|c_{n-h}\right|, \quad\left|a_{n}\right| \leqslant \frac{1}{\left[n+\lambda n(n-1)-\psi_{n}\right]} \sum_{h=1}^{n-1} \psi_{h}\left|a_{h}\right|\left|c_{n-h}\right| .
$$

Here by using Lemma 1.2 we get the following inequality

$$
\left|a_{n}\right| \leqslant \frac{\left|\delta_{k}\right|(A-B)}{\left[n+\lambda n(n-1)-\psi_{n}\right]} \sum_{h=1}^{n-1} \psi_{h}\left|a_{h}\right|
$$

Now we prove that

$$
\left|a_{n}\right| \leqslant \frac{\left|\delta_{k}\right|(A-B)}{2\left[n+\lambda n(n-1)-\psi_{n}\right]} \sum_{h=1}^{n-1} \psi_{h}\left|a_{h}\right| \leqslant \prod_{h=1}^{n-1} \frac{\left|\delta_{k}(A-B)-2\left(h-\psi_{h}+\lambda h(h-1)\right) B\right|}{2\left(h+1-\psi_{h+1}+\lambda h(h-1)\right)} .
$$


For this, we use the induction method. Putting $n=2$ in (2.5), we have

$$
\left|a_{2}\right| \leqslant \frac{\left|\delta_{k}\right|(A-B)}{\left[2+2 \lambda(2-1)-\psi_{2}\right]}=\frac{\left|\delta_{k}\right|(A-B)}{\left[2+2 \lambda-\psi_{2}\right]} .
$$

From (2.6) we have

$$
\left|a_{2}\right| \leqslant \frac{\left|\delta_{k}\right|(A-B)}{\left[2+2 \lambda-\psi_{2}\right]} \leqslant \prod_{h=1}^{n-1} \frac{\left|\delta_{k}(A-B)-2\left(1-\psi_{1}\right) B\right|}{2\left(2-\psi_{2}\right)} .
$$

Since $\psi_{1}=1$ we get

$$
\left|a_{2}\right| \leqslant \frac{\left|\delta_{k}(A-B)\right|}{2\left(2-\psi_{2}\right)}
$$

Putting $n=3$ in (2.5) we have

$$
\left|a_{3}\right| \leqslant \frac{\left|\delta_{k}\right|(A-B)}{2\left(3-\psi_{3}+3 \lambda(3-1)\right)}\left[1+\psi_{2}\left|a_{2}\right|\right], \quad\left|a_{3}\right| \leqslant \frac{\left|\delta_{k}\right|(A-B)}{2\left(3-\psi_{3}+6 \lambda\right)}\left[1+\psi_{2} \frac{\left|\delta_{k}\right|(A-B)}{2\left(2-\psi_{2}\right)}\right] .
$$

Now, from (2.6) we get

$$
\begin{aligned}
& \left|a_{3}\right| \leqslant\left[\frac{\left|\delta_{k}(A-B)\right|}{2\left(2-\psi_{2}+2 \lambda\right)}\right]\left[\frac{\left|\delta_{k}(A-B)-2\left(2-\psi_{2}+2 \lambda\right) B\right|}{2\left(3-\psi_{3}+6 \lambda\right)}\right], \\
& \left|a_{3}\right| \leqslant\left[\frac{\left|\delta_{k}\right|(A-B)}{2\left(2-\psi_{2}+2 \lambda\right)}\right]\left[\frac{\left|\delta_{k}\right|(A-B)+2\left(2-\psi_{2}+2 \lambda\right)}{2\left(3-\psi_{3}+6 \lambda\right)}\right], \\
& \left|a_{3}\right| \leqslant\left[\frac{\left|\delta_{k}\right|(A-B)}{2\left(3-\psi_{3}+6 \lambda\right)}\right]\left[\frac{\left|\delta_{k}\right|(A-B)}{2\left(2-\psi_{2}+2 \lambda\right)}+1\right] .
\end{aligned}
$$

Let the hypothesis be true for $n=m$, then from (2.5) we have

$$
\left|a_{m}\right| \leqslant \frac{\left|\delta_{k}\right|(A-B)}{2\left(m-\psi_{m}+\lambda m(m-1)\right)} \sum_{h=1}^{m-1} \psi_{h}\left|a_{h}\right|, \quad a_{1}=\psi_{1}=1 .
$$

From (2.6) we have

$$
\left|a_{m}\right| \leqslant \prod_{h=1}^{m-1} \frac{\left|\delta_{k}(A-B)-2\left(h-\psi_{h}+\lambda h(h-1)\right) B\right|}{2\left(h+1-\psi_{h+1}+\lambda h(h-1)\right)} .
$$

Using modulus properties we get

$$
\left|a_{m}\right| \leqslant \prod_{h=1}^{m-1} \frac{\left|\delta_{k}\right|(A-B)-2\left(h-\psi_{h}+\lambda h(h-1)\right)}{2\left(h+1-\psi_{h+1}+\lambda h(h-1)\right)} .
$$

By the induction hypothesis, we have

$$
\frac{\left|\delta_{k}\right|(A-B)}{2\left(m-\psi_{m}+\lambda m(m-1)\right)} \sum_{h=1}^{m-1} \psi_{h}\left|a_{h}\right| \leqslant \prod_{h=1}^{m-1} \frac{\left|\delta_{k}\right|(A-B)-2\left(h-\psi_{h}+\lambda h(h-1)\right)}{2\left(h+1-\psi_{h+1}+\lambda h(h-1)\right)} .
$$

Multiplying both sides of above expression by $\frac{\left|\delta_{k}\right|(A-B)+2\left(m-\psi_{m}+\lambda m(m-1)\right)}{2\left(m+1-\psi_{m+1}+\lambda m(m-1)\right)}$, we get

$$
\frac{\left|\delta_{k}\right|(A-B)+2\left(m-\psi_{m}+\lambda m(m-1)\right)}{2\left(m+1-\psi_{m+1}+\lambda m(m-1)\right)} \times \prod_{h=1}^{m-1} \frac{\left|\delta_{k}\right|(A-B)+2\left(h-\psi_{h}+\lambda h(h-1)\right)}{2\left(h+1-\psi_{h+1}+\lambda h(h-1)\right)}
$$




$$
\begin{aligned}
& \geqslant \frac{\left|\delta_{k}\right|(A-B)+2\left(m-\psi_{m}+\lambda m(m-1)\right)}{2\left(m+1-\psi_{m+1}+\lambda m(m-1)\right)} \times \frac{\left|\delta_{k}\right|(A-B)}{2\left(m-\psi_{m}+\lambda m(m-1)\right)} \sum_{h=1}^{m-1} \psi_{h}\left|a_{h}\right|, \\
\prod_{h=1}^{m} \frac{\left|\delta_{k}\right|(A-B)+2\left(h-\psi_{h}+\lambda h(h-1)\right)}{2\left(h+1-\psi_{h+1}+\lambda h(h-1)\right)} & \\
& \geqslant \frac{\left|\delta_{k}\right|(A-B)}{2\left(m-\psi_{m}+\lambda m(m-1)\right)}\left[\frac{\left|\delta_{k}\right|(A-B)+2\left(m-\psi_{m}+\lambda m(m-1)\right)}{2\left(m+1-\psi_{m+1}+\lambda m(m-1)\right)}\right] \sum_{h=1}^{m-1} \psi_{h}\left|a_{h}\right| \\
& =\frac{\left|\delta_{k}\right|(A-B)}{2\left(m+1-\psi_{m+1}+\lambda m(m-1)\right)}\left[\frac{\left|\delta_{k}\right|(A-B)}{2\left(m-\psi_{m}+\lambda m(m-1)\right)} \sum_{h=1}^{m-1} \psi_{h}\left|a_{h}\right|+\sum_{h=1}^{m-1} \psi_{h}\left|a_{h}\right|\right] \\
& \geqslant \frac{\left|\delta_{k}\right|(A-B)}{2\left(m+1-\psi_{m+1}+\lambda m(m-1)\right)}\left[\psi_{m}\left|a_{m}\right|+\sum_{h=1}^{m-1} \psi_{h}\left|a_{h}\right|\right] \\
& =\frac{\left|\delta_{k}\right|(A-B)}{2\left(m+1-\psi_{m+1}+\lambda m(m-1)\right)}\left[\sum_{h=1}^{m} \psi_{h}\left|a_{h}\right|\right] .
\end{aligned}
$$

Therefore,

$$
\frac{\left|\delta_{k}\right|(A-B)}{2\left(m+1-\psi_{m+1}+\lambda m(m-1)\right)}\left[\sum_{h=1}^{m} \psi_{h}\left|a_{h}\right|\right] \leqslant \prod_{h=1}^{m} \frac{\left|\delta_{k}\right|(A-B)+2\left(h-\psi_{h}+\lambda h(h-1)\right)}{2\left(h+1-\psi_{h+1}+\lambda h(h-1)\right)},
$$

which shows that the inequality is true for $n=m+1$. Hence (2.6) is true, i.e.,

$$
\left|a_{n}\right| \leqslant \frac{\left|\delta_{k}\right|(A-B)+2\left(h-\psi_{h}+\lambda h(h-1)\right)}{2\left(h+1-\psi_{h+1}+\lambda h(h-1)\right)},
$$

which completes the proof.

For $\lambda=0, j=i=1$ in above theorem we have the following results, proved by Noor and Malik [11].

Corollary 2.6. For $n \geqslant 2,-1 \leqslant B<A \leqslant 1, k \geqslant 0, f \in k-S T[A, B, N]$ is expressed in (1.1), then

$$
\left|a_{n}\right| \leqslant \prod_{j=0}^{n-2} \frac{\left|\delta_{k}(A-B)+2 j B\right|}{2(j+1)} .
$$

\section{Acknowledgment}

The work is supported by MOHE grant:FRGS/1/2016/STG06/UKM/01/1.

\section{References}

[1] F. S. M. Al Sarari, S. Latha, Conic regions and symmetric points, Int. J. Pure. Appl. Math., 97 (2014), 273-285. 2.1, 2.1

[2] F. S. M. Al Sarari, S. Latha, A note on coefficient inequalities for ( $j, i)$-symmetrical functions with conic regions, Bull. Int. Math. Virtual Inst., 6 (2016), 77-87. 1, 2

[3] A. W. Goodman, Open problems on univalent and multivalent functions, Bull. Amer. Math. Soc., 74 (1968), 1035-1050. 1

[4] W. Janowski, Some extremal problems for certain families of analytic functions, I, Ann. Polon. Math., 28 (1937), $297-326$. 1

[5] S. Kanas, Coefficient estimates in subclasses of the Carathodory class related to conical domains, Acta Math. Univ. Comenian. (N.S.), 74 (2005), 149-161. 1

[6] S. Kanas, A. Wiśniowska, Conic regions and k-uniform convexity, Continued fractions and geometric function theory (CONFUN), Trondheim, (1997), J. Comput. Appl. Math., 105 (1999), 327-336. 2.1, 2.1 
[7] S. Kanas, A. Wiśniowska, Conic domains and starlike functions, Rev. Roumaine Math. Pures Appl., 45 (2000), $645-658$. 1

[8] O. Kwon, Y.-J. Sim, A certain subclass of Janowski type functions associated with k-symmetric points, Commun. Korean Math. Soc., 28 (2013), 143-154. 1

[9] P. Liczberski, J. Poubiński, On (j, k)-symmetrical functions, Math. Bohem., 120 (1995), 13-28. 1, 1.1

[10] K. I. Noor, R. Fayyaz, M. A. Noor, Some classes of k-uniformly functions with bounded radius rotation, Appl. Math. Inf. Sci., 8 (2014), 527-533. 1

[11] K. I. Noor, S. N. Malik, On coefficient inequalities of functions associated with conic domains, Comput. Math. Appl., 62 (2011), 2209-2217. 1.2, 2.1, 2.1, 2.1

[12] Y. Polatoğlu, M. Bolcal, A. Şen, E. Yavuz, A study on the generalization of Janowski functions in the unit disc, Acta Math. Acad. Paedagog. Nyházi. (N.S.), 22 (2006), 27-31. 1 